\title{
A SECTORIAL THEOREM ON COMPLETELY RAMIFIED RATIONAL FUNCTIONS
}

\author{
ANDREAS SAUER \\ (Received 26 October 2006; revised 6 November 2007) \\ Communicated by P. C. Fenton
}

Abstract

We prove that under a sharp growth condition meromorphic functions posses a direction such that at most four rational functions are completely ramified in any sector containing the direction.

1991 Mathematics subject classification: 30D35.

Keywords and phrases: completely ramified, Julia direction, Ahlfors theory, filling discs, cercles de remplissages, singular direction.

\section{Introduction and statement of the theorem}

Let $f: \mathbb{C} \rightarrow \widehat{\mathbb{C}}$ be a transcendental meromorphic function. Then it follows from Nevanlinna's theory of meromorphic functions that the equation $f(z)=c$ has infinitely many solutions with at most two exceptional values $c \in \widehat{\mathbb{C}}$ (Picard's theorem) and that it has infinitely many simple solutions with at most four exceptional values $c \in \widehat{\mathbb{C}}$, so-called completely ramified values (see [4] or [16, Chapter 1]). The question whether the constants $c$ in the above equations can be replaced by more complicated objects, in particular by rational functions or so-called small functions for $f$, has a long history. As was shown by Nevanlinna [7, Chapter IV, p 39], Picard's theorem extends to the seemingly most general setting of small functions. From the generalization of Nevanlinna's second fundamental theorem stated in [14] it follows immediately that at most four small functions can be completely ramified.

Motivated by the results in sectorial value distribution theory (see [16]) we treat in this paper the following question: under which conditions does there exist a direction (a ray from 0 to $\infty$ ), such that at most four rational functions are completely ramified for $f$ in any sector containing the direction.

(C) 2008 Australian Mathematical Society 1446-7887/08 \$A2.00+0.00 
It is not very surprising that a theorem of this type holds, but it is also clear that such a direction cannot exist for every $f$ : there exist transcendental meromorphic functions that omit an open set of values around every direction. This can be shown by an analysis of an example given in [8] (see [13, Section 2]).

We show that the growth condition (1), given already in [9] in connection with a 'Picard version' of Theorem 1.1, is also sharp for our considerations.

The main result of this note is the following.

THEOREM 1.1. Let $f: \mathbb{C} \rightarrow \widehat{\mathbb{C}}$ be a transcendental meromorphic function with

$$
\limsup _{r \rightarrow \infty} \frac{T(r, f)}{(\log r)^{3}}=\infty .
$$

Then there exists a direction such that, for any distinct rational functions $\varphi_{1}, \ldots, \varphi_{5}$, in every sector containing that direction at least one of the equations $f=\varphi_{i}$ has infinitely many simple solutions.

The growth condition (1) is sharp in the following sense: in [9] an example was given with $T(r, f) \sim \frac{1}{3}(\log r)^{3}$ such that, around every direction, $f$ omits infinitely many rational functions, which are then of course completely ramified.

It is worth noting that a direction with at most four completely ramified constants exists already if in (1) the denominator $(\log r)^{3}$ is replaced by $(\log r)^{2}$. This is also a sharp growth condition, as follows easily from the results in [5, 8] and [11, Theorem 4.1.4].

In order to prove Theorem 1.1 we collect some facts from value distribution theory and prove several lemmata in the next section. We note that this line of thought, with minor adjustments, reproves the fact that a transcendental meromorphic function (without any growth restriction) has at most four completely ramified rational functions. (See the remark at the end of the paper.)

\section{Value distribution theory and some preliminaries}

If $D \subset \widehat{\mathbb{C}}$ is a domain and $f: \mathbb{C} \rightarrow \widehat{\mathbb{C}}$ is meromorphic, then the bounded components of $f^{-1}(D)$ are called the islands of $f$ over $D$. If $I$ is an island over $D$ then $f: I \rightarrow D$ is a proper map and its degree is called the multiplicity of the island. If $I$ has multiplicity 1 , that is if $f$ is one-to-one on $I$, then $I$ is called a simple island.

The following lemma is an easy consequence of Rouché's theorem. It constitutes the basic idea of our method (see also [10]). As usual $\mathbb{D}_{\delta}(0)$ denotes the open disc of radius $\delta$ around 0 .

LEMMA 2.1. Let $f: \mathbb{C} \rightarrow \widehat{\mathbb{C}}$ be meromorphic with infinitely many simple islands $I_{n}$ over $\mathbb{D}_{\delta}(0)$ and $\varphi$ be rational with $\varphi(\infty)=0$. Then $f-\varphi$ has infinitely many simple zeros in $\cup I_{n}$. 
The rest of this section deals with the fact that there need not exist simple islands over $\varphi(\infty)$ and that many target functions $\varphi$ can have the same value at infinity. We develop a statement that works in simply connected islands with an additional property (Lemma 2.6).

The following theorem is one of the main consequences of Ahlfors's theory of covering surfaces [1].

THEOREM 2.2. Let $f: \mathbb{C} \rightarrow \widehat{\mathbb{C}}$ be a non-constant meromorphic function and $D_{1}, \ldots, D_{q} \subset \widehat{\mathbb{C}}$ be simply connected domains with disjoint closures. If every simply connected island of $f$ over $D_{i}$ has multiplicity at least $\mu_{i}$ then

$$
\sum_{i=1}^{q}\left(1-\frac{1}{\mu_{i}}\right) \leq 2 .
$$

An application of Zalcman's rescaling lemma [17] to Theorem 2.2 shows the following.

COROLLARY 2.3. Let $\mathcal{F}$ be a non-normal family of meromorphic functions on $\mathbb{D}$ and $D_{1}, \ldots, D_{q} \subset \widehat{\mathbb{C}}$ be simply connected domains with disjoint closures.

If $q=3$ and if all but finitely many $f \in \mathcal{F}$ have no simple island over any of the $D_{i}$ then there are two $D_{i}$ such that, with at most finitely many exceptions, all $f \in \mathcal{F}$ have simply connected islands over both $D_{i}$.

If $q=4$ and if all but finitely many $f \in \mathcal{F}$ have no simple island over any of the $D_{i}$ then, with at most finitely many exceptions, all $f \in \mathcal{F}$ have simply connected islands over every $D_{i}$.

If $q=5$ then there exists at least one $D_{i}$, such that, with at most finitely many exceptions, all $f \in \mathcal{F}$ have simple islands over this $D_{i}$.

For the proof one first has to enlarge the $D_{1}, \ldots, D_{q} \subset \widehat{\mathbb{C}}$ slightly (e.g. using polygonal paths surrounding the domains) such that Ahlfors's theory can still be applied to the limit function obtained from Zalcman's lemma with respect to the larger domains. A Rouché argument then yields the statement. The statement for $q=5$ is also known as the Ahlfors five islands theorem.

The next lemma follows from the Riemann-Hurwitz formula in the form treated in [12]. Here $r(I):=\sum_{z \in I}\left(\right.$ mult $\left._{f}(z)-1\right)$ denotes the ramification of $f$ on $I$, where mult $_{f}(z)$ is the multiplicity of $f$ at $z$.

LEMMA 2.4. Let I and D be simply connected domains and $f: I \rightarrow D$ be a proper holomorphic map. Then

$$
\operatorname{deg} f=1+r(I)
$$

The following lemma makes it possible to work in simply connected islands. A value $z_{0} \in \widehat{\mathbb{C}}$ is called completely ramified for a meromorphic function $f$, if all preimages $f^{-1}\left(z_{0}\right)$ have multiplicity at least 2 . 
LEMMA 2.5. Let $I$ and $D$ be simply connected domains, $z_{0} \in D$ and $f: I \rightarrow D$ be a proper holomorphic map such that $z_{0}$ is completely ramified. Further, let $\varphi: I \rightarrow D$ be holomorphic such that $\overline{\varphi(I)}$ is contained in a simply connected domain $G$ with $\bar{G} \subset D \backslash\left\{z_{0}\right\}$. Then $f-\varphi$ has at least two simple zeros in $I$.

Proof. Let $d:=\operatorname{deg} f$. The preimage $f^{-1}(G)$ consists of $q \leq d$ components $U_{1}, \ldots, U_{q}$ which are simply connected. (If $G$ is a disc then the $U_{i}$ are simply connected by the maximum principle. For the general case use the Riemann mapping theorem.) Let $d_{i}$ be the degree of $f: U_{i} \rightarrow G$ and $r\left(U_{i}\right)$ be the ramification of $f$ on $U_{i}$. Then application of Lemma 2.4 on each $U_{i}$ and summation gives

$$
d-q=\sum_{i=1}^{q} r\left(U_{i}\right)
$$

Clearly $d=\left|f^{-1}\left(\left\{z_{0}\right\}\right)\right|+r\left(f^{-1}\left(\left\{z_{0}\right\}\right)\right)$ (where $|\cdot|$ denotes cardinality). Since $z_{0}$ is completely ramified, $\left|f^{-1}\left(\left\{z_{0}\right\}\right)\right| \leq d / 2$ and $r\left(f^{-1}\left(\left\{z_{0}\right\}\right)\right) \geq d / 2$. From Lemma 2.4 it follows that

$$
d-1=r(I)
$$

so that $\sum_{i=1}^{q} r\left(U_{i}\right) \leq r(I)-r\left(f^{-1}\left(\left\{z_{0}\right\}\right)\right) \leq d / 2-1$. This and (3) show that $q \geq d / 2+1$. Now suppose that $r\left(U_{i}\right) \geq 1$ for at least $q-1$ of the domains $U_{i}$. Then

$$
r(I) \geq r\left(f^{-1}\left(\left\{z_{0}\right\}\right)\right)+\sum_{i=1}^{q} r\left(U_{i}\right) \geq \frac{d}{2}+q-1 \geq d .
$$

This contradicts (4). Hence, we can assume $r\left(U_{i}\right)=0$ for $i=1,2$, which implies that $U_{1}, U_{2}$ are simple islands of $f$ over $G$.

If $G$ is a disc with centre $z_{1}$ then by assumption $\left|\varphi-z_{1}\right|<\left|f-z_{1}\right|$ on $\partial U_{i}$, $i=1,2$. By Rouché's theorem $f-\varphi$ and $f-z_{1}$ both have exactly one simple zero in $U_{1}$ and $U_{2}$, which proves the lemma in this case. For the general situation use the Riemann mapping theorem.

For $I \subset \mathbb{C}$, which is assumed to be contained in a proper sub-sector of $\mathbb{C}$, we set

$$
\Delta \arg (I):=\max _{z, w \in I}|\arg z-\arg w| .
$$

LEMMA 2.6. Let $f: \mathbb{C} \rightarrow \widehat{\mathbb{C}}$ be meromorphic, with infinitely many simply connected islands $I_{n}$ over $\mathbb{D}_{\delta}(0)$ with $\Delta \arg \left(I_{n}\right) \rightarrow 0$, and let $\varphi_{1}, \varphi_{2}$ be distinct rational functions with $\varphi_{1}(\infty)=\varphi_{2}(\infty)=0$. Then for at least one $k=1,2$ it follows that $f-\varphi_{k}$ has infinitely many simple zeros in $\cup I_{n}$.

PROOF. Suppose that $f-\varphi_{2}$ possesses only finitely many simple zeros in $\cup I_{n}$. Consider $\widetilde{f}:=f-\varphi_{2}$ and $\widetilde{\varphi}:=\varphi_{1}-\varphi_{2}$ and a sequence $\widetilde{I}_{n} \subset I_{n}$ of islands of $\widetilde{f}$ over $\mathbb{D}_{\delta / 2}(0)$. Such $\widetilde{I}_{n}$ exist since $\varphi_{2}(\infty)=0$. Further, all $\widetilde{I}_{n}$, with at most finitely many exceptions, are simply connected, because a bounded component of the complement 
of such an island contains a pole of $\widetilde{f}$. Except for finitely many, these have to be poles of $f$, which is impossible since $\tilde{\widetilde{I}}_{n} \subset I_{n}$ and the $I_{n}$ are simply connected. For each of the proper mappings $\tilde{f}: \widetilde{I}_{n} \rightarrow \mathbb{D}_{\delta / 2}(0)$ the value $z_{0}=0$ is completely ramified. Further $\tilde{\varphi}(z)=(c+o(1)) z^{-k}$ as $z \rightarrow \infty$ for some $c \neq 0$ and $k \in \mathbb{N}$. For $n$ large enough we have by assumption $\Delta \arg \left(\tilde{I}_{n}\right)<2 \pi / k$. Increasing $n$ if necessary we obtain $\Delta \arg \left(\widetilde{\varphi}\left(\tilde{I}_{n}\right)\right)<2 \pi$. Further $d|z|^{-k}<|\widetilde{\varphi}(z)|<D|z|^{-k}$ for some $d, D>0$ for large $z$. It follows that for $\tilde{\varphi}: \widetilde{I}_{n} \rightarrow \mathbb{D}_{\delta / 2}(0)$ there exist $l_{n}, L_{n}>0$ such that for $n \geq n_{0}$

$$
\overline{\widetilde{\varphi}\left(\widetilde{I_{n}}\right)} \subset\left\{w \in \mathbb{D}_{\delta / 2}(0)\left|l_{n}<\right| w \mid<L_{n}<\delta / 2, \arg w \in\left(x_{n}, y_{n}\right)\right\}=: G_{n},
$$

with $y_{n}-x_{n}<2 \pi . G_{n}$ is a simply connected domain with $\overline{G_{n}} \subset \mathbb{D}_{\delta / 2}(0) \backslash\{0\}$. We can apply Lemma 2.5 to $\widetilde{f}, \widetilde{\varphi}: \widetilde{I}_{n} \rightarrow \mathbb{D}_{\delta / 2}(0)$, which proves the claim.

\section{Proof of Theorem 1.1}

In [9, Lemmas 2.2 and 2.3] it was proved that if (1) holds, then $f$ has a sequence of $\operatorname{discs} \mathbb{D}_{j}:=\left\{z \in \mathbb{C}|| z-z_{j}\left|<\alpha_{j}\right| z_{j} \mid\right\}$ with $\alpha_{j} \rightarrow 0$ that forms a sequence of filling discs (or cercles de remplissage) for every Möbius transformation of $f$ with rational functions as coefficients. We work exclusively in these discs. A sequence of discs $\mathbb{D}_{j}$ as described above is a sequence of filling discs if $f$ takes on every infinite union $\cup \mathbb{D}_{j_{k}}$ all values in $\widehat{\mathbb{C}}$ with at most two exceptions infinitely often. It follows immediately from [9, the proof of Theorem 1.2] that we can choose the sequence $\alpha_{j} \rightarrow 0$ such that the functions

$$
f_{j}: \mathbb{D} \rightarrow \mathbb{D}_{j}, \quad f_{j}(z):=f\left(z_{j}+\alpha_{j} z_{j} z\right),
$$

form a non-normal family in $\mathbb{D}$. Hence, we can use Corollary 2.3. Further, we may assume that $\arg z_{j} \rightarrow \beta$, so that the ray $t e^{i \beta}$ with $t>0$ will be a suitable direction.

Let $\varphi_{1}, \ldots, \varphi_{5}$ be distinct rational functions. Note that we may apply Möbius transformations with rational functions as coefficients to $f$ and all $\varphi_{i}$ without affecting the assertion. Set $M:=\left\{\varphi_{i}(\infty) \mid i=1, \ldots, 5\right\}$ and denote by $a_{k}$ the elements of $M$. Further, we define

$$
T(w, z):=\frac{w-\varphi_{1}(z)}{w-\varphi_{2}(z)} \cdot \frac{\varphi_{3}(z)-\varphi_{2}(z)}{\varphi_{3}(z)-\varphi_{1}(z)} .
$$

Considering $T(f(z), z)$ instead of $f$ and $T\left(\varphi_{i}(z), z\right)$ instead of $\varphi_{i}$ we may assume that $\{0,1, \infty\} \subset M$ and hence $|M| \geq 3$. Choose discs $\mathbb{D}_{\delta}\left(a_{k}\right)$ around the values of $M$ that have disjoint closures. We say that $f$ has islands over $a_{k}$ if $f$ has infinitely many islands in $\cup \mathbb{D}_{j}$ over the disc containing $a_{k}$.

If $|M|=5$ the assertion follows from Lemma 2.1 and Corollary 2.3 with $q=5$.

Suppose $|M|=4$. If there are infinitely many simple islands over one of these four values, then the claim follows again from Lemma 2.1. Hence, according to 
Corollary 2.3 with $q=4$, we can assume that there are infinitely many simply connected islands over each value in $M$. Two $\varphi_{i}$ have the same value at $\infty$, say $\varphi_{4}(\infty)=\varphi_{5}(\infty)$. Applying a Möbius transformation we may assume $\varphi_{4}(\infty)=0$ and $\varphi_{5}(\infty)=0$. Since $\Delta \arg \left(\mathbb{D}_{j}\right) \rightarrow 0$, Lemma 2.6 proves the assertion in this case.

We now assume $|M|=3$. Using (5) we may suppose that $\varphi_{1} \equiv 0, \varphi_{2} \equiv \infty$ and $\varphi_{3} \equiv 1$. Assume that over none of the values $\{0,1, \infty\}$ are there infinitely many simple islands.

(i) Suppose there are two values in $M$ such that for both values there are two $\varphi_{i}$ taking the value at $\infty$. Then we can argue exactly as in the case $|M|=4$, since by Corollary 2.3 with $q=3$ over at least one of the two values there are infinitely many simply connected islands.

(ii) If without loss of generality $\varphi_{4}(\infty)=\varphi_{5}(\infty)=\infty$ then both functions are nonconstant since they are distinct from $\varphi_{2}$. We can assume that $c:=\left(\varphi_{4} / \varphi_{5}\right)(\infty) \neq$ 0 . Then apply the transformation $w / \varphi_{5}$. (This means that (5) was not a good choice. Instead one should take

$$
T(w, z):=\frac{w-\varphi_{1}(z)}{w-\varphi_{2}(z)} \cdot \frac{\varphi_{5}(z)-\varphi_{2}(z)}{\varphi_{5}(z)-\varphi_{1}(z)},
$$

from the start.)

We get $\varphi_{1} \equiv 0, \varphi_{2} \equiv \infty, \varphi_{3}(\infty)=0, \varphi_{4}(\infty)=c$ and $\varphi_{5} \equiv 1$. Depending on $c$ this leads to (i) or to the case $|M|=4$.

\section{Remarks}

(a) It is remarkable that the growth condition (1) was already used in [15] to prove the existence of Hayman directions. Hayman directions are singular directions corresponding to Hayman's classical theorem [4, Corollary to Theorem 3.5], on the value distribution of $f$ and its derivatives: $f$ takes every value of $\mathbb{C}$ infinitely often or every derivative $f^{(k)}$ takes every value in $\mathbb{C} \backslash\{0\}$ infinitely often in any sector containing the direction. In the proofs of the results given in $[9,15]$ and Theorem 1.1 one can take the same filling discs. This leads to the following theorem.

THEOREM 4.1. Let $f: \mathbb{C} \rightarrow \widehat{\mathbb{C}}$ be a transcendental meromorphic function satisfying (1). Then there exists a Julia direction for $f$ that is a Hayman direction, where at most two rational functions are omitted and where at most four rational functions are completely ramified.

It is unclear whether (1) is needed for the existence of Hayman directions. If the Hayman direction is supposed to be also a Julia direction which is generated by filling discs, which is implicit in the proof of [15], then again the example in [9] shows that (1) is sharp. (We refer to [2] and [3].) 
(b) Our method can be used to prove that every transcendental meromorphic function has at most four completely ramified rational functions. In the proof of Theorem 1.1 it was important that the filling discs stay filling discs after application of transformations with rational functions as coefficients. This was assured by (1). For functions of slow growth it may happen that the transformation leads to (i) filling discs in other places or even (ii) no filling discs at all. Point (i) is of no importance for the proof of a non-sectorial theorem. Problem (ii) occurs if the transformed function is a so-called Julia exceptional function (see [8]). This is equivalent to $f^{\#}(z)=O(1 /|z|)$ for $z \rightarrow \infty$ where $f^{\#}=\left|f^{\prime}\right| /\left(1+|f|^{2}\right)$ is the spherical derivative of $f$. Let $z_{j} \rightarrow \infty$ be a sequence such that $\liminf _{j \rightarrow \infty}\left|z_{j}\right| f^{\#}\left(z_{j}\right) \geq 1 / 2$. Such a sequence exists by a theorem of Lehto and Virtanen [5, 6]. From the Marty criterion it easily follows that the sequence $f_{j}(z):=f\left(z_{j} z\right)$ is normal in $\mathbb{C} \backslash\{0\}$. Hence there exists a subsequence converging to some function $F$ meromorphic in $\mathbb{C} \backslash\{0\}$. Since $F^{\#}(1)=\lim _{k \rightarrow \infty}\left|z_{j_{k}}\right| f^{\#}\left(z_{j_{k}}\right) \geq 1 / 2$ it follows that $F$ is non-constant. Suppose that $|M|=3$. Then $F$ takes at least two of the $a_{k}$ or has a simple root for one $a_{k}$. This follows from Theorem 2.2 applied to the function $F$ o exp. Let $z_{1}$ be a root of $F$ for some $a_{k}$. It is not complicated to check that $\delta$ can be chosen so that $z_{1}$ is contained in a simply connected island $I$ over $\mathbb{D}_{2 \delta}\left(a_{k}\right)$ with $I \subset \mathbb{D}_{\eta}\left(z_{1}\right)$, where $\eta$ is chosen such that $\Delta \arg \left(\mathbb{D}_{\eta}\left(z_{1}\right)\right)<\varepsilon$. In addition, if $z_{1}$ is a simple root then $\delta$ can be chosen such that $I$ is a simple island. It follows that $f$ has appropriate islands over $\mathbb{D}_{\delta}\left(a_{k}\right)$ with 'small' $\Delta$ arg in the discs $z_{j_{k}} \mathbb{D}_{\eta}\left(z_{1}\right)$, so that the proof of Lemma 2.6 also works in this situation. The case $|M|=4$ can be treated analogously.

\section{Acknowledgements}

I would like to thank W. Bergweiler and A. Eremenko for interesting discussions about completely ramified functions.

\section{References}

[1] L. V. Ahlfors, 'Zur Theorie der Überlagerungsflächen', Acta Math. 65 (1935), 157-194.

[2] P. C. Fenton and J. Rossi, 'Cercles de remplissage for entire functions', Bull. London Math. Soc. 31 (1999), 59-66.

[3] P. C. Fenton and J. Rossi, 'Growth of functions in cercles de remplissage', J. Aust. Math. Soc. 72 (2002), 131-136.

[4] W. K. Hayman, Meromorphic functions (Oxford University Press, London, 1975).

[5] O. Lehto, 'The spherical derivative of meromorphic functions in the neighbourhood of an isolated singularity', Comment. Math. Helv. 33 (1959), 196-205.

[6] O. Lehto and K. I. Virtanen, 'On the behaviour of meromorphic functions in the neighbourhood of an isolated singularity', Ann. Acad. Sci. Fenn. Ser. A. I. 240 (1957).

[7] R. Nevanlinna, Le théorème de Picard-Borel et a théorie des fonctions méromorphes (GauthierVillars, Paris, 1939). Reprint Chelsea, New York, 1974.

[8] A. Ostrowski, 'Über Folgen analytischer Funktionen und einige Verschärfungen des Picardschen Satzes', Math. Z. 24 (1924), 215-258.

[9] J. Rossi, 'A sharp result concerning cercles de remplissage', Ann. Acad. Sci. Fenn. Ser. A I 20 (1995), 179-185. 
[10] A. Sauer, 'Deficient rational functions and Ahlfors's theory of covering surfaces', Ark. Mat. 39 (2001), 151-155.

[11] J. L. Schiff, Normal families (Springer, Berlin, 1993).

[12] N. Steinmetz, 'The formula of Riemann-Hurwitz and iteration of rational functions', Complex Var. Theory Appl. 22 (1993), 203-206.

[13] N. Toda, 'Sur les directions de Julia et de Borel des fonctions algebroides', Nagoya Math. J. 34 (1969), 1-23.

[14] K. Yamanoi, 'The second main theorem for small functions and related problems', Acta Math. 192 (2004), 225-294.

[15] L. Yang, 'Meromorphic functions and their derivatives', J. London Math. Soc. 25 (1982), $288-296$.

[16] L. Yang, Value distribution theory (Springer, Berlin, 1993).

[17] L. Zalcman, 'Normal families: new perspectives', Bull. Amer. Math. Soc. 35 (1998), 215-230.

ANDREAS SAUER, FH Dortmund, University of Applied Sciences and Arts, Sonnenstr. 96, 44047 Dortmund, Germany

e-mail: andreas.sauer@fh-dortmund.de 